ISSN 2519-7398 (Versión electrónica)

DOI: http://dx.doi.org/10.21704/ac.v78i2.1066

(C) Universidad Nacional Agraria La Molina, Lima - Perú

\title{
Método sistemático para obtener las curvas de caudal en una bomba hidraulica artesanal usado en el sector agricola y pecuaria: Basado en simulacion y programacion vectorial
}

\author{
A Systematic method for obtaining flow curves in a craft hydraulic pump used in the agricultural \\ and livestock sector: Based on simulation and vector programming
}

\author{
Alexis Enrique Rubio Valle ${ }^{1}$
}

*Autor de correspondencia

\begin{abstract}
Resumen
Las necesidades de agua en el sector agrícola y pecuaria son un aspecto crítico de la producción. El proceso productivo agrícola y pecuaria, prácticamente dependen de la obtención y el traslado del líquido elemento a las zonas de producción agrícola o bebedero de los animales. Las bombas de construcción artesanal son utilizadas para este fin, en el presente trabajo estudiaremos una bombas de pistón de construcción artesanal a través de un método sistemático que permite analizar todo el ciclo de trabajo de la bomba hasta lograr la curva de caudales y otros resultados. Con el método sistemático planteado aquí, tomando como datos únicamente las revoluciones de la manivela, provenientes de una fuente de movimiento, (hélices o motor) y las longitudes de los elementos del sistema biela-manivela, se obtiene el movimiento lineal del pistón representándose en curvas de caudales de agua. Este trabajo contiene un desarrollo sistemático, a través del modelamiento, programación, simulación de los mecanismos y las leyes que gobierna la transformación del movimiento. La importancia de este estudio radica en ofrecer mayor luz y precisión en el análisis del mecanismo de transformación de movimiento, que se realiza en una bomba eólica de construcción artesanal, esto permite, por un lado precisar la cuantificación de la cantidad de agua ofrecida por la bomba y por otro lado ver los efectos que producen el modificar la longitud de algún elemento en el mecanismo, pudiendo optimizar el sistema para las distintas necesidades de agua generadas por los proceso agrícolas o pecuarios. Los objetivos son establecer un método sistemático para la obtención de la productividad de la bomba, así como obtener las curvas de caudales - ángulo de posición de la manivela, caudal - tiempo, para velocidades angulares de 3,6,9 RPM a la manivela. Los resultados ofrecen Tablas de solución al análisis del movimiento relativo, en los que se encuentran los valores para la velocidad relativa del elemento biela y la velocidad absoluta del pistón, la cual están representadas en curvas de velocidades, además de las curvas de caudales de succión y bombeo por unidad de tiempo y por ángulo de posición de la manivela.
\end{abstract}

Palabras claves: Método Sistemático, Caudal, Bomba, Eólica, Movimiento Relativo, Programación, Vectores, Transformación, Mecanismos, Biela, Manivela, Simulación, Succión, Descarga, Ángulo, Posición, Proceso.

\begin{abstract}
Water needs in the agricultural and livestock sector are a critical aspect of production. The agricultural production process and livestock, practically depend on the obtaining and the transfer of liquid element to the areas of agricultural production or watering of the animals. Handmade construction pumps are used for this purpose, in this work, we will study a piston pump of handmade construction through a systematic method that allows to analyze the whole work cycle of the pump until reaching the flow and other results curve. With the systematic method proposed here, taking as data only the crank revolutions from a source of motion (propellers or motor) and the lengths of the elements of the crank-crank system, the linear motion of the piston is obtained by representing in Water flow curves. This work contains a systematic development, through modeling, programming, and simulation of mechanisms and laws that governs the transformation of movement. The importance of this study is to offer greater light and precision in the analysis of the mechanism of transformation of movement, which is carried out in a wind farm handcrafted, this allows, on the one hand, to quantify the quantity of water offered by the Pump and on the other hand to see the effects that modify the length of some element in the mechanism, being able to optimize the system for the different water needs generated by the agricultural or livestock processes. The objectives are to establish a systematic method to obtain the productivity of the pump, as well as to obtain the flow curves - crank position angle, flow - time, for angular velocities of 3, 6, 9 RPM to the crank. The results provide tables of solution to the relative motion analysis, in which the values for the relative velocity of the connecting rod element and the absolute speed of the piston, which are represented in velocity curves, in addition
\end{abstract}

${ }^{1}$ Facultad de Ingeniería Agrícola, Universidad Nacional Agraria La Molina, Apartado postal 12-056 - La Molina, Lima, Perú. Email: arubiov@ lamolina.edu.pe 
to the suction flow curves And pumping per unit time and by crank position angle.

Keywords: Systematic Method; Flow; Pump; Wind; Movement; Relative; Programming; Vectors; Transformations; Mechanisms; Connecting rod; Crank; Simulation; Suction; Discharge; Angle; Position; Process.

\section{Introducción}

Las estadísticas del Ministerio de Agricultura (MINAG) indican que en el Perú alrededor de la tercera parte de la población vive en las zonas rurales, cuyos ingresos dependen del $50 \%$ de la agricultura, además se estima que el área de riego en el país está representado por un $70 \%$ bajo secano, $26.9 \%$ bajo riego por gravedad, $2 \%$ bajo riego tecnificado y $0,7 \%$ a través de la explotación de las aguas subterráneas mediante pozos. Frente a esta situación, con el apoyo de universidades y organizaciones internacionales, se vienen desarrollando e implementando proyectos de riego usando energías renovables, para esto se analizan los diferentes tipos de energía que se pueden usar para cada situación y lugar específico. Se ha hecho investigación para la implementación de grandes aerobombas en el afán de extraer agua del subsuelo, pero se ha hecho poco en el análisis de bombas de bajas revoluciones y construcción artesanal., En el presente trabajo nos vamos a centrar en la bombas de pistón alternativas de construcción artesanal, en las cuales el rotor eólico esta acoplado mecánicamente a una bomba de pistón a través de un vástago que transmite el movimiento oscilante desde la parte superior de la torre hasta la bomba sumergida generalmente dentro del pozo o fuente de agua. Este sistema, es por lejos, la más común en las soluciones de aerobombeo al nivel mundial (Figura 1).

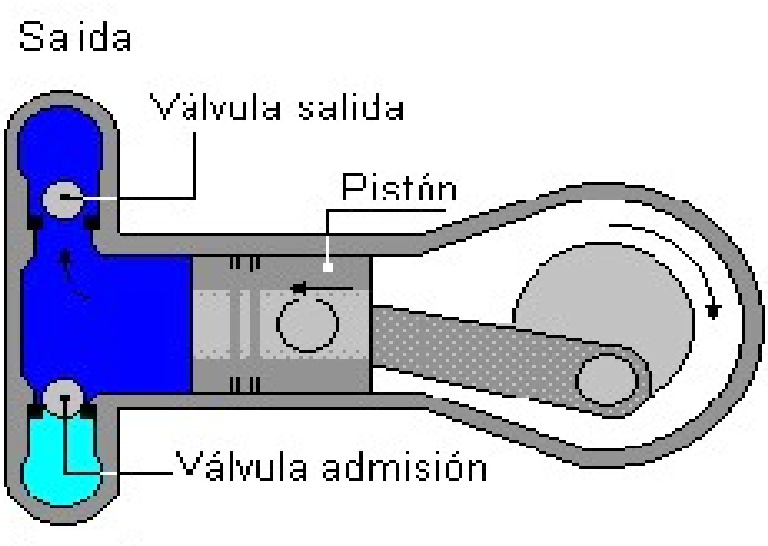

Entrada

Figura 1. Bomba reciprocante

Este sistema es ideal para ser aplicado a sistemas de bombeo de bajo caudal o construcción artesanal, por ser un mecanismo relativamente sencillo y de bajo costo, la complejidad reside en el análisis de transformación de movimiento, tanto para fuerzas como para velocidades y aceleraciones. Este cálculo se realiza en base al tema de movimientos relativos en el plano, Figura 2. (J. Meriam 1995) (R.C. Hibbeler 2005).
El estudio se justifica en el hecho que existe muy poca aplicación sistemática al modelamiento, programación y simulación de movimiento en el estudio de los mecanismos de transferencia de movimiento para la obtención de los caudales finales ofrecidos por una bomba eólica de construcción artesanal, solo se calcula el caudal promedio ofrecido por este sistema.

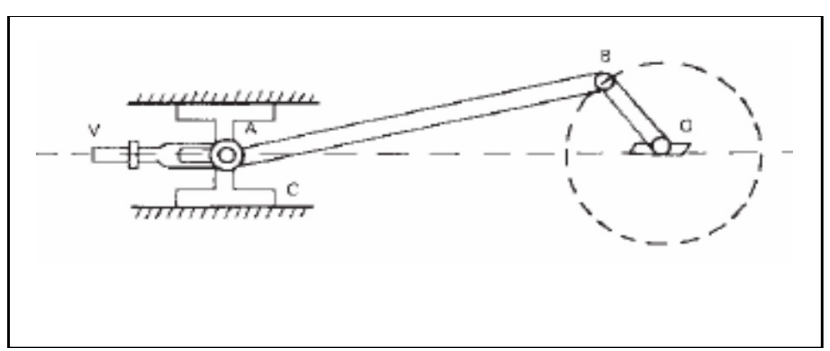

Figura 2. Idealización del mecanismo Biela-Manivela

Este estudio es importante porque hoy en día la manera en que se obtienen los datos de caudal en una bomba eólica de construcción casera, se obtienen en forma muy rudimentaria, se llenan volúmenes de agua y se mide el tiempo, de esta manera se obtiene el caudal promedio de producción de la bomba, pero esto no es preciso ni mucho menos científico, La importancia del método radica en que únicamente con las revoluciones de entrada y las longitudes de las barras del sistema, se puede obtener las curvas de caudal. Además esto es, si es que la bomba ya esta construida, pero a través de este método se puede simular, solo con los datos mencionados, lo que entregaría la bomba si estuviera construida, es decir la predicción de los resultados antes de su construcción, comparándose si es esa la necesidad de agua, o habría que modificar la bomba, Una importancia adicional seria que en estas simulaciones se pueden hacer análisis de sensibilidad, modificando las longitudes de los elementos y observando el efecto del caudal medido. He ahí lo importante de sistematizar este conocimiento a través de programación y simulación como los utilizados en este estudio (Solid Work, Geogebra). El presente estudio se ha realizado para una bomba eólica de bajas revoluciones, esto es un máximo de $53 \mathrm{~cm}^{3} / \mathrm{seg}$. a una velocidad de rotación proveniente de las hélices de 3, 6, y 9 RPM como velocidades angulares constantes de trabajo a la manivela.

\section{Materiales y métodos}

Los materiales utilizados fueron un molino de viento de construcción casera, presente en el campus de la Universidad Nacional Agraria La Molina. Instrumentos de medición: como wincha de seis metros, pie de rey, escuadra de $30 \mathrm{~cm}$, transportador, alicates, juego de llaves de medida 
además de las llaves stilson y francesa, Programa de digitalización en 3 D "SOLID WORK", Programa de simulación en 2D "GEOGEBRA", hoja de cálculo EXEL, útiles de escritorio en ingeniería.

\section{Métodos utilizados}

\section{Método: análisis de máquina simple}

"Una máquina simple (Charles De Coulomb, 1736-1806) es un dispositivo que transforma en trabajo útil la fuerza aplicada, el trabajo de entrada se realiza mediante la aplicación de una sola fuerza, y la máquina realiza el trabajo de salida a través de otra fuerza única, El mecanismo de biela-manivela "permite convertir el movimiento giratorio continuo de un eje en uno lineal alternativo en el pie de la biela". El sistema biela-manivela emplea, básicamente, una manivela, un soporte y una biela cuya cabeza se conecta con el eje excéntrico de la manivela (empuñadura) Figura 3. El eje dispone de un movimiento giratorio que transmite a la manivela. La manivela convierte el movimiento giratorio del eje en uno circular en su empuñadura (eje excéntrico). La cabeza de la biela está unida a la empuñadura de la manivela (eje excéntrico) y por tanto, está dotada de un movimiento circular, Figura 3. En su movimiento circular, la cabeza de la biela arrastra el pie de biela, que sigue un movimiento lineal alternativo. La trayectoria seguida por el pie de biela es lineal alternativa.Figura 4

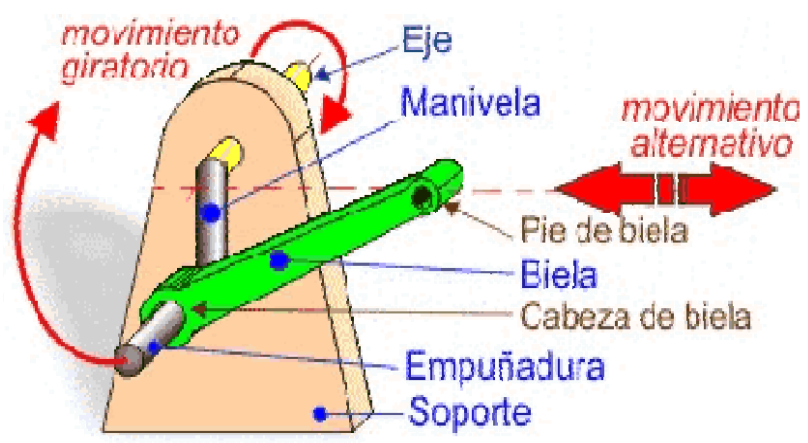

Figura 3. Representación del mecanismo Biela-Manivela

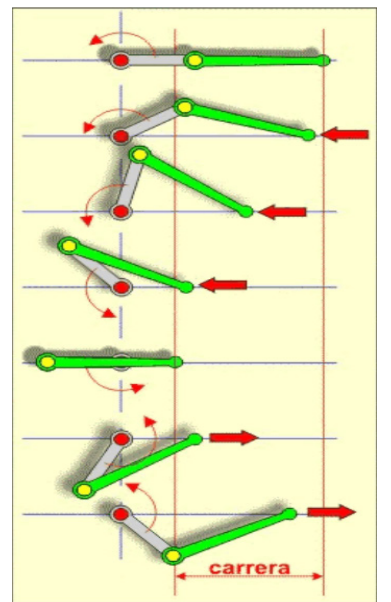

Figura 4. Funcionamiento mecanismo Biela- Manivela
Estos principios físicos a utilizar lo realiza el estudio de la dinámica del cuerpo rígido (F.P. Beer ; Dinámica), (J. Meriam; Dinámica),

\section{Principio de las velocidades relativas - método gráfico}

Es común utilizarlo en cursos de ingeniería para obtener la velocidad de una barra, pistón u otros elementos de un mecanismo. Si se acepta que las distancias entre los diversos puntos de un sólido no varían mientras este se está moviendo por el espacio, entonces el sólido es modelizable como sólido rígido y conocida la velocidad angular del sólido en cada instante y la velocidad de un punto $\mathrm{O}$ del sólido, podemos conocer la velocidad de cualquier punto $\mathrm{P}$ a través del triangulo vectorial de velocidades (Figura 5).

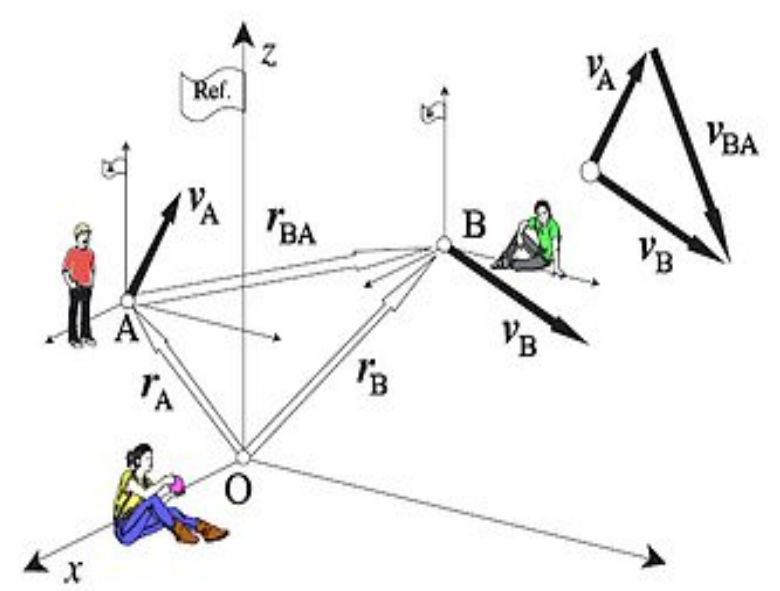

Figura 5. Principio de las velocidades relativas y el triángulo vectorial de velocidades

\section{El método sistemático}

Es un proceso mediante el cual se relacionan hechos aparentemente aislados y se formula un concepto que unifica los diversos elementos. Consiste en la reunión racional de varios elementos dispersos en una nueva totalidad, siendo esta la idea formulada al inicio del presente trabajo, Esta sistematización formulada se representa de la siguiente manera.

- Sistemas CAD-CAM de diseño (Programa Solid Work).- Con el que se digitaliza los datos tomados en campo de la bomba eólica, se modelan los elementos, se ensamblan virtualmente y se simula el movimiento físico de la bomba y sus mecanismos en tiempo real, como un todo. (Figura 6)

- Programa Geogebra para análisis vectorial cuantitativo,. se diseña el programa con las longitudes y la geometría del sistema en su conjunto, se generan los triángulos de velocidades, (movimiento relativo), y se simula el movimiento de la manivela. dando como resultado el movimiento del pistón. 

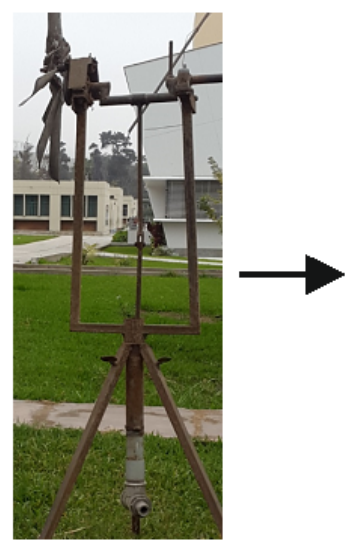

Bomba de

construcción

artesanal UNALM
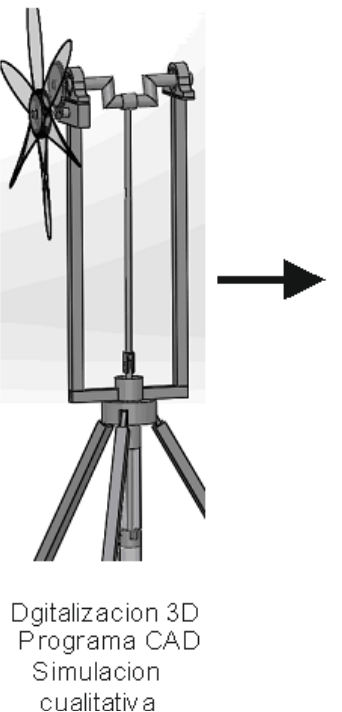

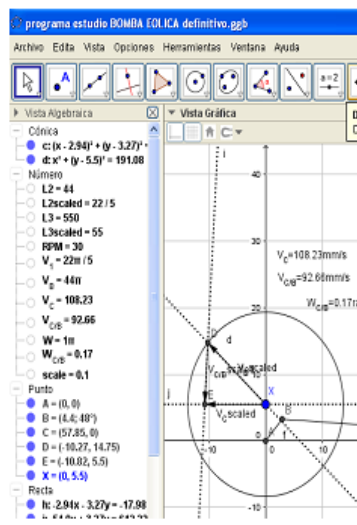

Programacion y simulacion de movimiento cuantitativo

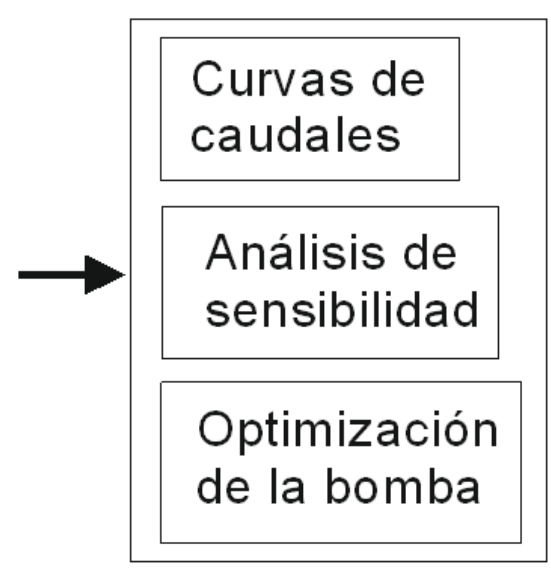

Resultados del método sistemático

Figura 6. Método sistemático para el análisis de una bomba de construcción artesanal

\section{Metodología de estudio}

Se realizó la toma de datos del molino de viento, en la Universidad Agraria La Molina, mecanismos como las hélices, el sistema de engranajes reductores, el mecanismo Biela - Manivela, las dimensiones del pistón y del cilindro. La digitalización a escala real de la bomba eólica, con sus mecanismos mencionados, utilizando un sistema CAD como lo es el Solid Work, para el análisis real del proceso de transferencia de movimiento desde la entrada de las velocidades de rotación en el rotor de la hélice, hasta el movimiento lineal en el pistón, Una vez culminado, se realiza la simulación de movimiento en 3D. Con la geometría (de los mecanismos se realiza el análisis cinemático, basados en los principios del movimiento relativo (J. Meriam; Dinámica) para cada $10^{\circ}$ de rotación de la manivela, lo que exige el análisis de 36 puntos de posición y la solución de 72 ecuaciones tomadas en sistemas de dos en dos. Se realizó la programación en vectores para la simulación vectorial en el programa Geogebra. Teniendo como datos las velocidades de rotación de la manivela, la geometría del sistema relativo (biela - manivela) y los puntos de posición y de tiempo en el momento de análisis. Compilación y procesamiento de datos de las simulaciones en hojas de calculo EXEL debidamente ordenadas. Esto da como resultado Tablas de soluciones para los parámetros como la velocidad relativa de la biela y las velocidades absolutas del pistón las cuales generan los caudales por cada punto de análisis. Se realizan las graficas para las curvas de resultados; Velocidad - Ángulo de posición, Caudal - Ángulo de posición, Velocidad - tiempo, caudal - tiempo,

\section{Resultados y discusión}

En las tablas se muestran de resultados cada $10^{\circ}$ y en las Figuras 7, 8, 9, y 10) se ven la velocidad del pistón $\mathrm{V}_{\mathrm{C}}$, la velocidad relativa de la biela $\mathrm{V}_{\mathrm{C} / \mathrm{B}}$ y la velocidad angular de la biela $\mathrm{W}_{\mathrm{C} / \mathrm{B}}$.
Tabla 1. Programación en vectores. Velocidad de rotación de la manivela 3 RPM

\begin{tabular}{|c|c|c|c|c|}
\hline ANG $\left(^{\circ}\right)$ & $\mathrm{V}\left(\mathrm{cm}^{3}\right)$ & t (s) & $\mathrm{V}(\mathrm{mm} / \mathrm{s})$ & $\mathrm{Q}\left(\mathrm{mm}^{3} / \mathrm{s}\right)$ \\
\hline 0 & 0.00 & 0.00 & 0.00 & 0.00 \\
\hline 10 & 1.19 & 0.56 & 2.59 & 3253.43 \\
\hline 20 & 4.70 & 1.11 & 5.08 & 6387.49 \\
\hline 30 & 10.40 & 1.67 & 7.39 & 9287.80 \\
\hline 40 & 18.06 & 2.22 & 9.43 & 11850.09 \\
\hline 50 & 27.38 & 2.78 & 11.14 & 13992.65 \\
\hline 60 & 38.00 & 3.33 & 12.45 & 15646.39 \\
\hline 70 & 49.55 & 3.89 & 13.35 & 16771.08 \\
\hline 80 & 61.61 & 4.44 & 13.80 & 17345.36 \\
\hline 90 & 73.79 & 5.00 & 13.82 & 17370.49 \\
\hline 100 & 85.70 & 5.56 & 13.42 & 16867.84 \\
\hline 110 & 97.00 & 6.11 & 12.63 & 15875.10 \\
\hline 120 & 107.37 & 6.67 & 11.49 & 14440.02 \\
\hline 130 & 116.55 & 7.22 & 10.04 & 12620.41 \\
\hline 140 & 124.33 & 7.78 & 8.34 & 10480.35 \\
\hline 150 & 130.55 & 8.33 & 6.43 & 8082.69 \\
\hline 160 & 135.07 & 8.89 & 4.37 & 5494.02 \\
\hline 170 & 137.81 & 9.44 & 2.21 & 2778.42 \\
\hline 180 & 138.73 & 10.00 & 0.00 & 0.00 \\
\hline 190 & 137.81 & 10.56 & -2.21 & -2778.42 \\
\hline 200 & 135.07 & 11.11 & -4.37 & -5494.02 \\
\hline 210 & 130.55 & 11.67 & -6.43 & -8082.69 \\
\hline 220 & 124.33 & 12.22 & -8.34 & -10480.35 \\
\hline 230 & 116.55 & 12.78 & -10.04 & -12620.41 \\
\hline 240 & 107.37 & 13.33 & -11.49 & -14440.02 \\
\hline 250 & 97.00 & 13.89 & -12.63 & -15875.10 \\
\hline 260 & 85.70 & 14.44 & -13.42 & -16867.84 \\
\hline 270 & 73.79 & 15.00 & -13.82 & -17370.49 \\
\hline 280 & 61.61 & 15.56 & -13.80 & -17345.36 \\
\hline 290 & 49.55 & 16.11 & -13.35 & -16771.08 \\
\hline 300 & 38.00 & 16.67 & -12.45 & -15646.39 \\
\hline 310 & 27.38 & 17.22 & -11.14 & -13992.65 \\
\hline 320 & 18.06 & 17.78 & -9.43 & -11850.09 \\
\hline
\end{tabular}


Continuation table 1

\begin{tabular}{ccccc}
\hline 330 & 10.40 & 18.33 & -7.39 & -9287.80 \\
340 & 4.70 & 18.89 & -5.08 & -6387.49 \\
350 & 1.19 & 19.44 & -2.59 & -3253.43 \\
$\mathbf{3 6 0}$ & $\mathbf{0 . 0 0}$ & $\mathbf{2 0 . 0 0}$ & $\mathbf{0 . 0 0}$ & $\mathbf{0 . 0 0}$ \\
\hline
\end{tabular}

Tabla 2. Programación en vectores (Programa Geogebra). Velocidad de rotación de la manivela 6 RPM

\begin{tabular}{|c|c|c|c|c|}
\hline $\operatorname{ANG}\left(^{\circ}\right)$ & $\mathrm{v}\left(\mathrm{cm}^{3}\right)$ & $\mathrm{t}(\mathrm{s})$ & $\mathrm{V}(\mathrm{mm} / \mathrm{s})$ & $\mathrm{Q}\left(\mathrm{mm}^{3} / \mathrm{s}\right)$ \\
\hline 0 & 0.00 & 0.00 & 0.00 & 0.00 \\
\hline 10 & 1.19 & 0.28 & 5.18 & 6508.12 \\
\hline 20 & 4.70 & 0.56 & 10.17 & 12776.23 \\
\hline 30 & 10.40 & 0.83 & 14.78 & 18574.35 \\
\hline 40 & 18.06 & 1.11 & 18.86 & 23701.43 \\
\hline 50 & 27.38 & 1.39 & 22.27 & 27984.05 \\
\hline 60 & 38.00 & 1.67 & 24.90 & 31292.78 \\
\hline 70 & 49.55 & 1.94 & 26.69 & 33542.16 \\
\hline 80 & 61.61 & 2.22 & 27.61 & 34689.47 \\
\hline 90 & 73.79 & 2.50 & 27.65 & 34740.99 \\
\hline 100 & 85.70 & 2.78 & 26.85 & 33736.94 \\
\hline 110 & 97.00 & 3.06 & 25.27 & 31750.19 \\
\hline 120 & 107.37 & 3.33 & 22.98 & 28880.03 \\
\hline 130 & 116.55 & 3.61 & 20.09 & 25242.07 \\
\hline 140 & 124.33 & 3.89 & 16.68 & 20960.71 \\
\hline 150 & 130.55 & 4.17 & 12.87 & 16166.64 \\
\hline 160 & 135.07 & 4.44 & 8.74 & 10988.03 \\
\hline 170 & 137.81 & 4.72 & 4.42 & 5556.85 \\
\hline 180 & 138.73 & 5.00 & 0.00 & 0.00 \\
\hline 190 & 137.81 & 5.28 & -4.42 & -5556.85 \\
\hline 200 & 135.07 & 5.56 & -8.74 & -10988.03 \\
\hline 210 & 130.55 & 5.83 & -12.87 & -16166.64 \\
\hline 220 & 124.33 & 6.11 & -16.68 & -20960.71 \\
\hline 230 & 116.55 & 6.39 & -20.09 & -25242.07 \\
\hline 240 & 107.37 & 6.67 & -22.98 & -28880.03 \\
\hline 250 & 97.00 & 6.94 & -25.27 & -31750.19 \\
\hline 260 & 85.70 & 7.22 & -26.85 & -33736.94 \\
\hline 270 & 73.79 & 7.50 & -27.65 & -34740.99 \\
\hline 280 & 61.61 & 7.78 & -27.61 & -34689.47 \\
\hline 290 & 49.55 & 8.06 & -26.69 & -33542.16 \\
\hline 300 & 38.00 & 8.33 & -24.90 & -31292.78 \\
\hline 310 & 27.38 & 8.61 & -22.27 & -27984.05 \\
\hline 320 & 18.06 & 8.89 & -18.86 & -23701.43 \\
\hline 330 & 10.40 & 9.17 & -14.78 & -18574.35 \\
\hline 340 & 4.70 & 9.44 & -10.17 & -12776.23 \\
\hline 350 & 1.19 & 9.72 & -5.18 & -6508.12 \\
\hline 360 & 0.00 & 10.00 & 0.00 & 0.00 \\
\hline
\end{tabular}

El análisis de maquina simple para un mecanismo biela manivela, es un problema sumamente complejo que además del análisis cinemático expresado en los resultados, se encuentran otras consideraciones, como lo expresa Mejia Balseca K. E. 1986 en su tesis de grado. El expresa lo siguiente: "El volumen entregado depende del área transversal, el tiempo, la carrera, las vueltas por minuto del cigüeñal, el numero de émbolos y las perdidas, estas se originan con la rapidez con que se cierran las válvulas, variando entre 0.94 y 0.98 ". Los resultados obtenidos
Tabla 3. Programación en vectores (Programa Geogebra). Velocidad de rotación de la manivela 9 RPM

\begin{tabular}{|c|c|c|c|c|}
\hline $\operatorname{ANG}\left({ }^{\circ}\right)$ & $\mathrm{v}\left(\mathrm{cm}^{3}\right)$ & $\mathrm{t}(\mathrm{s})$ & $\mathrm{V}(\mathrm{mm} / \mathrm{s})$ & $\mathrm{Q}\left(\mathrm{mm}^{3} / \mathrm{s}\right)$ \\
\hline 0 & 0.00 & 0.00 & 0.00 & 0.00 \\
\hline 10 & 1.19 & 0.19 & 7.77 & 9761.56 \\
\hline 20 & 4.70 & 0.37 & 15.25 & 19163.72 \\
\hline 30 & 10.40 & 0.56 & 22.17 & 27862.16 \\
\hline 40 & 18.06 & 0.74 & 28.29 & 35551.52 \\
\hline 50 & 27.38 & 0.93 & 33.40 & 41976.70 \\
\hline 60 & 38.00 & 1.11 & 37.35 & 46939.16 \\
\hline 70 & 49.55 & 1.30 & 40.04 & 50311.98 \\
\hline 80 & 61.61 & 1.48 & 41.41 & 52034.83 \\
\hline 90 & 73.79 & 1.67 & 41.47 & 52111.48 \\
\hline 100 & 85.70 & 1.85 & 40.27 & 50604.77 \\
\hline 110 & 97.00 & 2.04 & 37.90 & 47622.77 \\
\hline 120 & 107.37 & 2.22 & 34.47 & 43320.05 \\
\hline 130 & 116.55 & 2.41 & 30.13 & 37862.47 \\
\hline 140 & 124.33 & 2.59 & 25.02 & 31441.06 \\
\hline 150 & 130.55 & 2.78 & 19.30 & 24249.33 \\
\hline 160 & 135.07 & 2.96 & 13.12 & 16483.31 \\
\hline 170 & 137.81 & 3.15 & 6.63 & 8336.53 \\
\hline 180 & 138.73 & 3.33 & 0.00 & 0.00 \\
\hline 190 & 137.81 & 3.52 & -6.63 & -8336.53 \\
\hline 200 & 135.07 & 3.70 & -13.12 & -16483.31 \\
\hline 210 & 130.55 & 3.89 & -19.30 & -24249.33 \\
\hline 220 & 124.33 & 4.07 & -25.02 & -31441.06 \\
\hline 230 & 116.55 & 4.26 & -30.13 & -37862.47 \\
\hline 240 & 107.37 & 4.44 & -34.47 & -43320.05 \\
\hline 250 & 97.00 & 4.63 & -37.90 & -47625.29 \\
\hline 260 & 85.70 & 4.81 & -40.27 & -50604.77 \\
\hline 270 & 73.79 & 5.00 & -41.47 & -52111.48 \\
\hline 280 & 61.61 & 5.19 & -41.41 & -52034.83 \\
\hline 290 & 49.55 & 5.37 & -40.04 & -50311.98 \\
\hline 300 & 38.00 & 5.56 & -37.35 & -46939.16 \\
\hline 310 & 27.38 & 5.74 & -33.40 & -41976.70 \\
\hline 320 & 18.06 & 5.93 & -28.29 & -35551.52 \\
\hline 330 & 10.40 & 6.11 & -22.17 & -27862.16 \\
\hline 340 & 4.70 & 6.30 & -15.25 & -19163.72 \\
\hline 350 & 1.19 & 6.48 & -7.77 & -9761.56 \\
\hline 360 & 0.00 & 6.67 & 0.00 & 0.00 \\
\hline
\end{tabular}

consideran todos los factores mencionados, no se estudian las perdidas por ser un análisis puramente cinemático del mecanismo sin considerar aun dentro del análisis, el mecanismo ni el tipo de válvulas en la bomba. Seria de mayor precisión aumentar una columna en los resultados afectándolo por el factor mencionado.

Los resultados se han obtenido aplicando el método grafico para la solución de ecuaciones vectoriales como lo expresa Meriam J. 1995 en su texto de Dinámica. pero, al igual que la mayoría de autores en textos avanzados de enseñanza en Ingeniería, estos hacen el análisis de puntos instantáneos de tiempo y posición en forma aislada, (Mabie, H. 1978. Beer, F. 2010), lo cual se complementa en el presente estudio con la sistematización del proceso, obteniéndose no solo soluciones puntuales, si no simulaciones de movimiento, Tablas y curvas de caudales. 

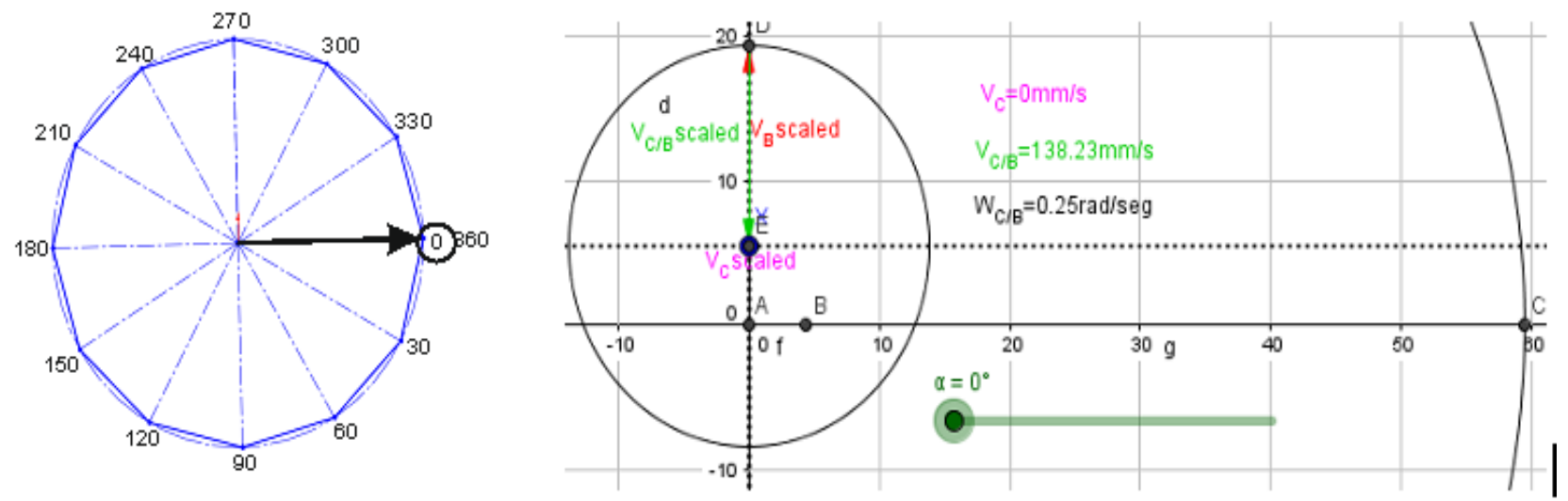

Figura 7. Representación del ángulo de posición $0^{\circ}$ de la manivela
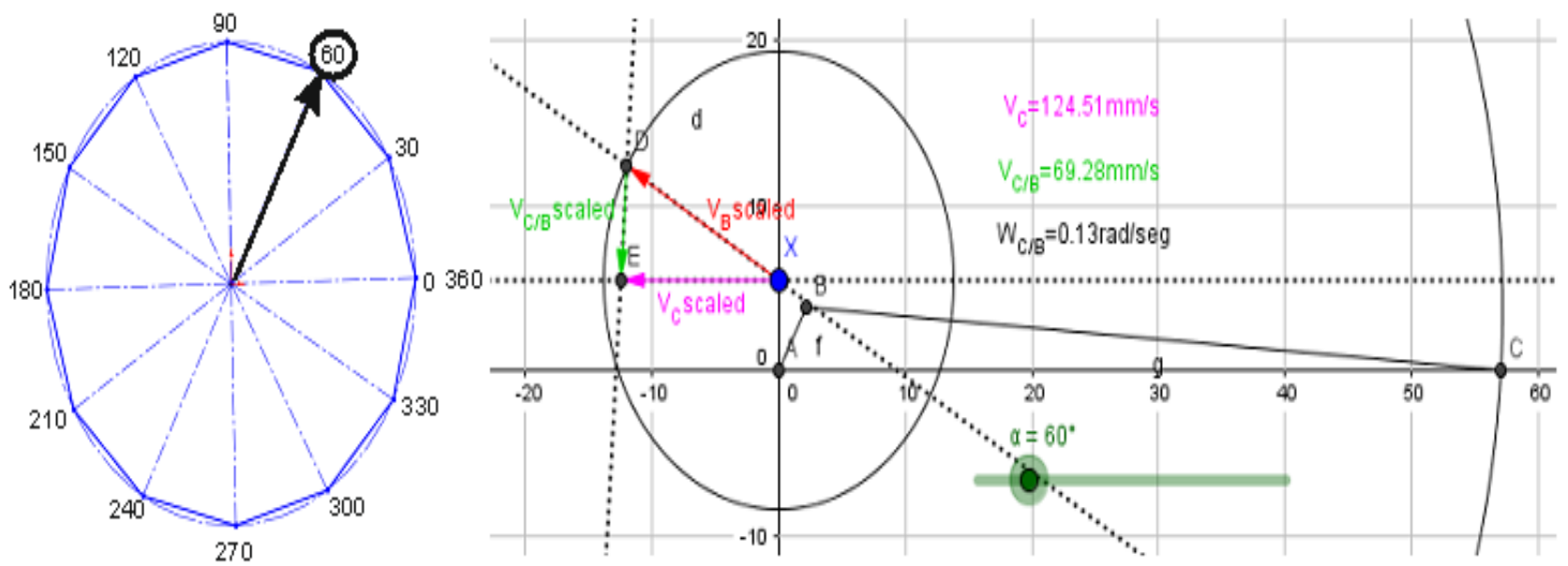

Figura 8. Representación del ángulo de posición $60^{\circ}$ de la manivela
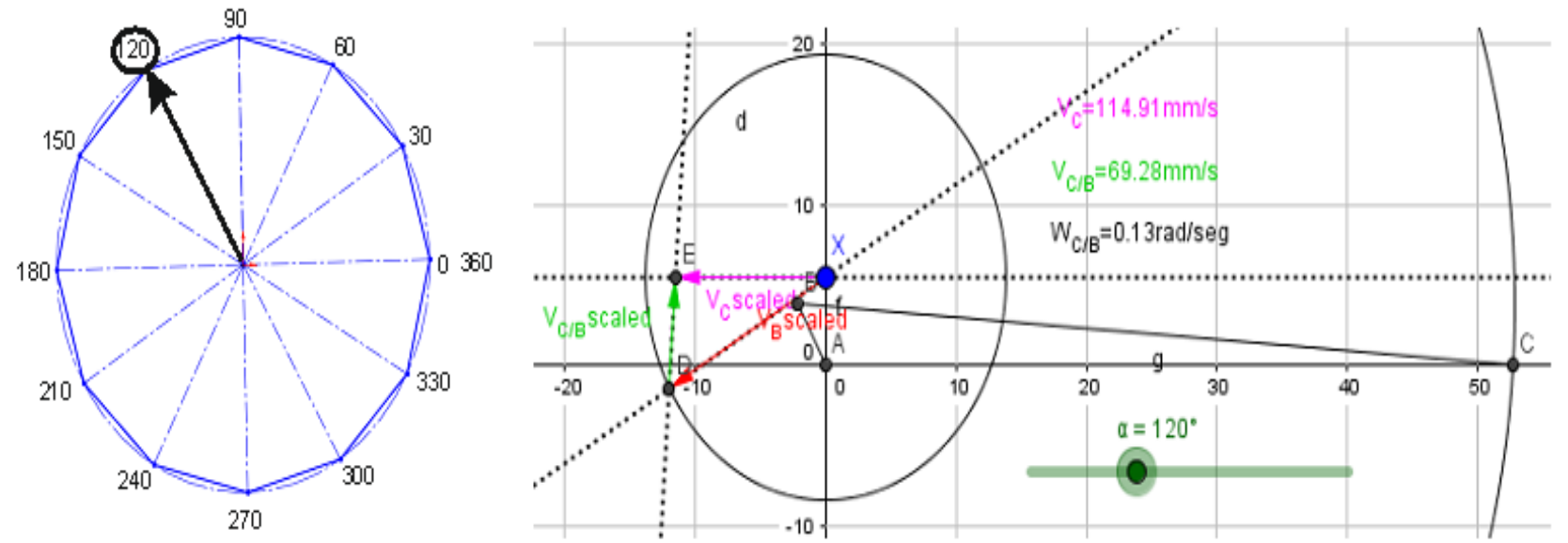

Figura 9. Representación del ángulo de posición $120^{\circ}$ de la manivela 

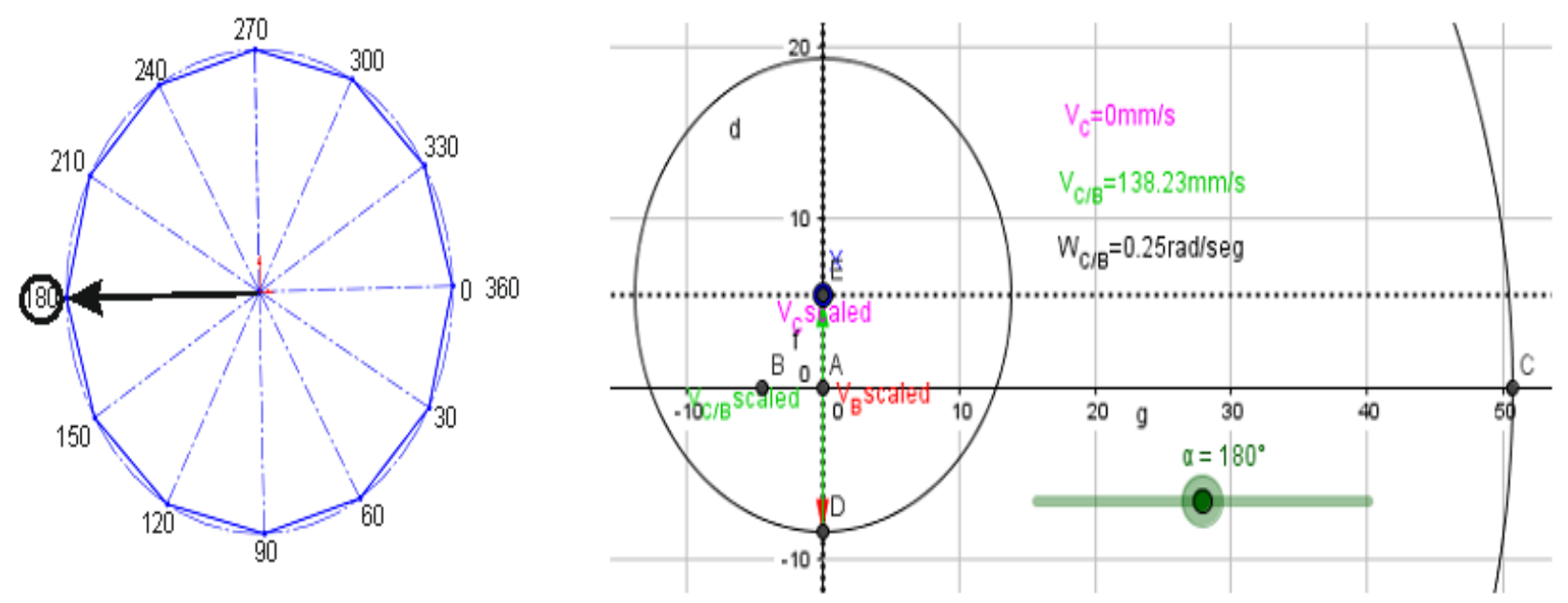

Figura 10. Representación del ángulo de posición $180^{\circ}$ de la manivela

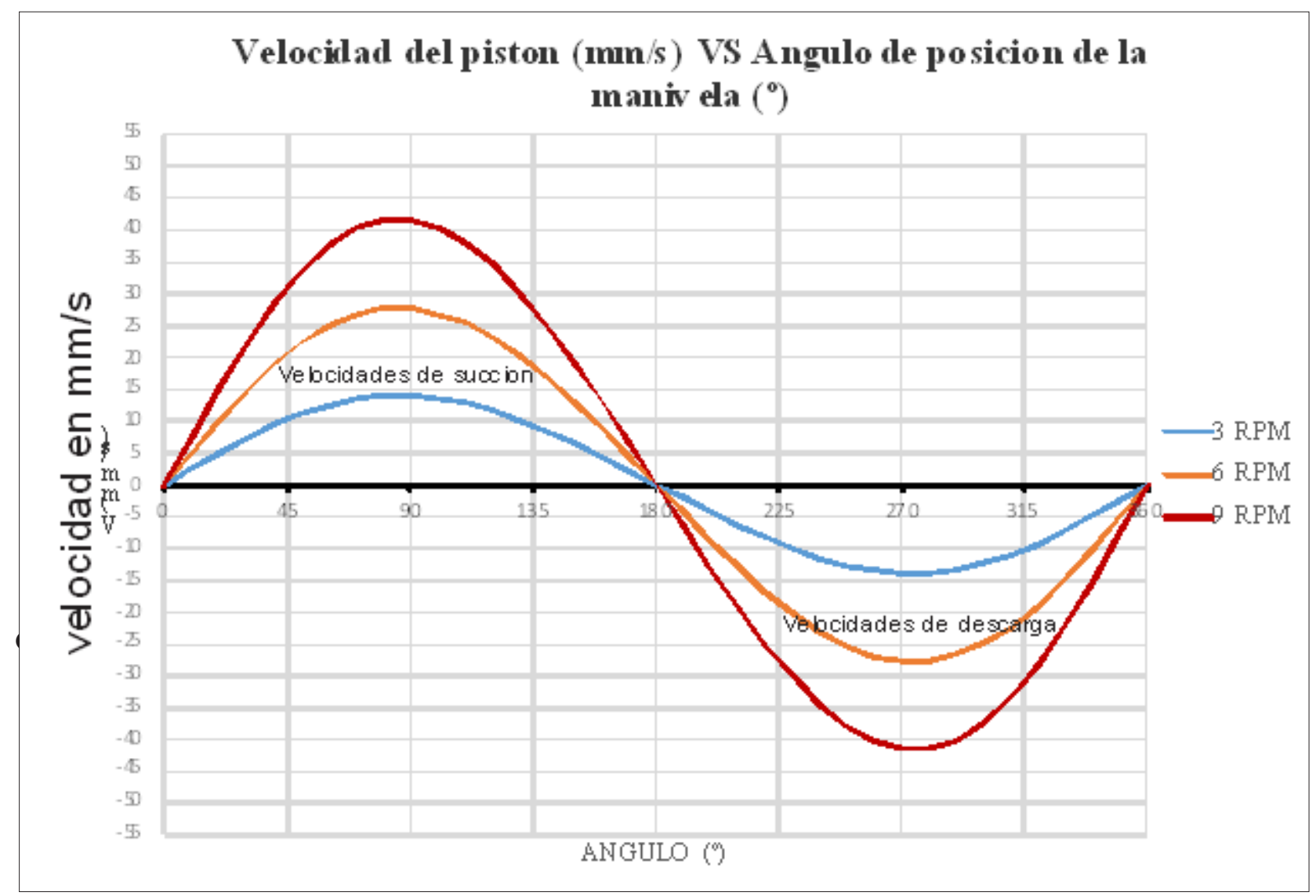

Figura 11. Variaciones en la velocidad del pistón, según el ángulo de posición de la manivela 


\section{C aud al (mm3/s) VS Angul o de posicion de la maniv ed a $\left({ }^{\circ}\right)$}

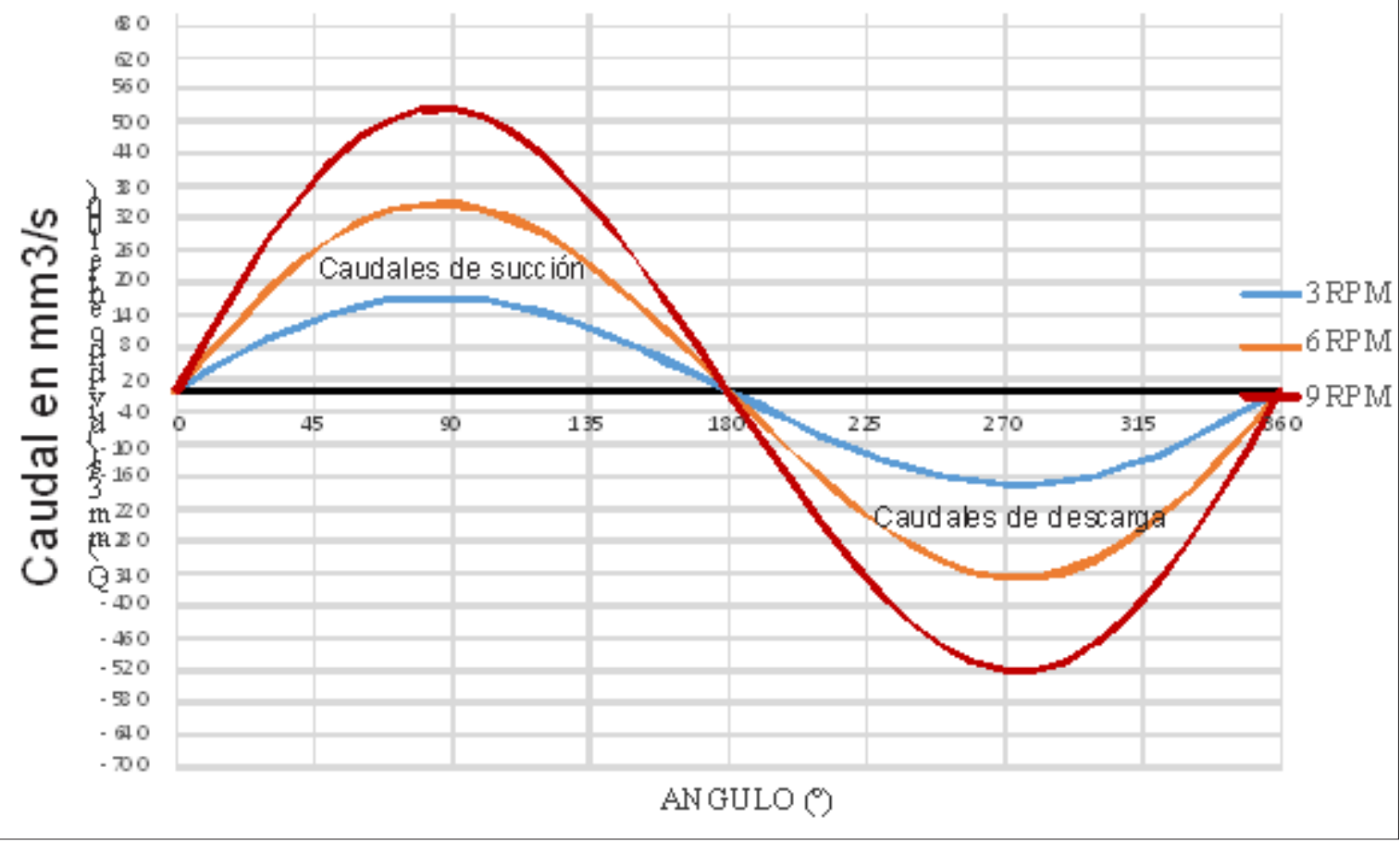

Figura 12. Variaciones en el caudal de la bomba, según el ángulo de posición de la manivela

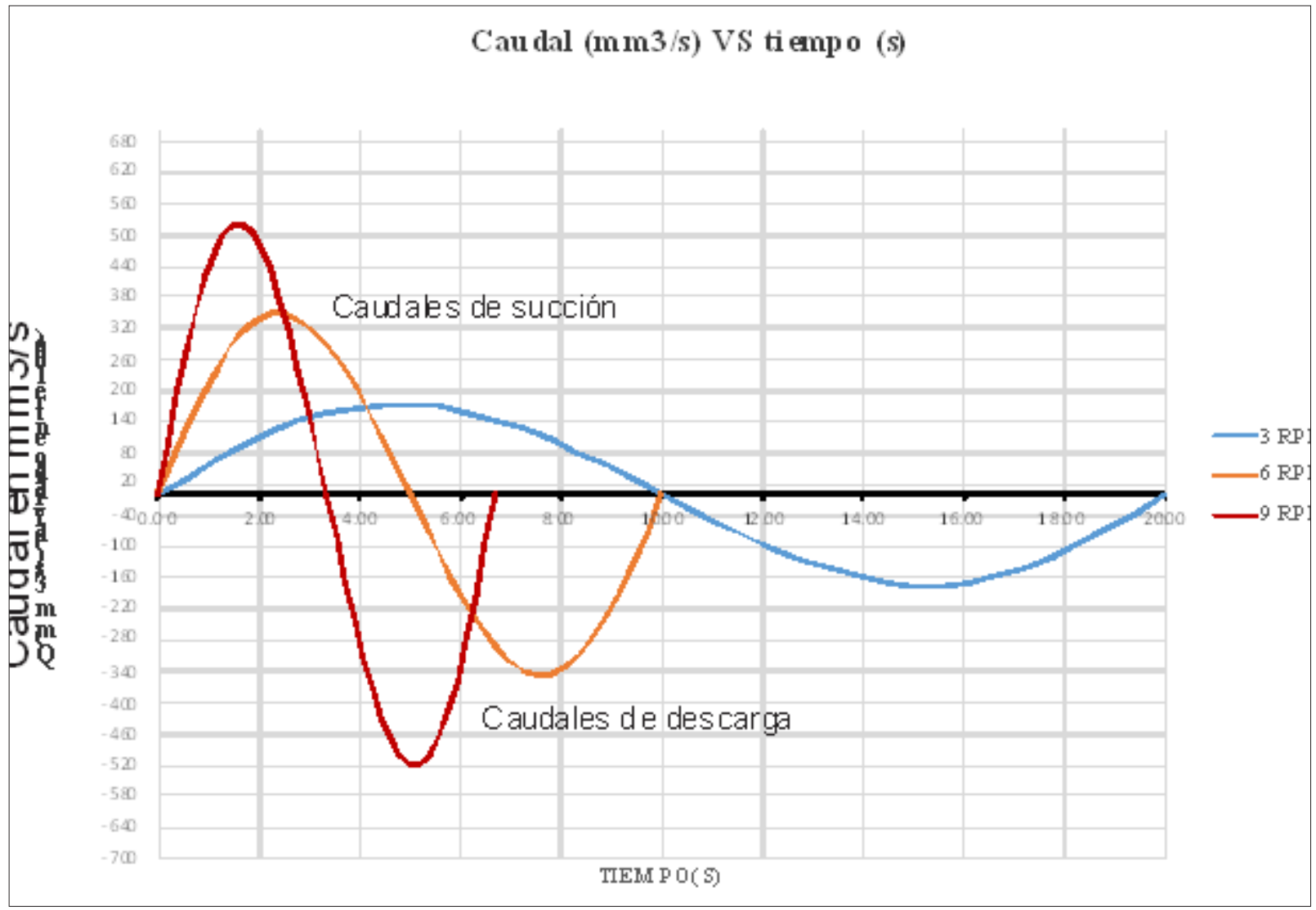

Figura 13. Variaciones en caudal de la bomba, según el ángulo de posición de la Manivela 


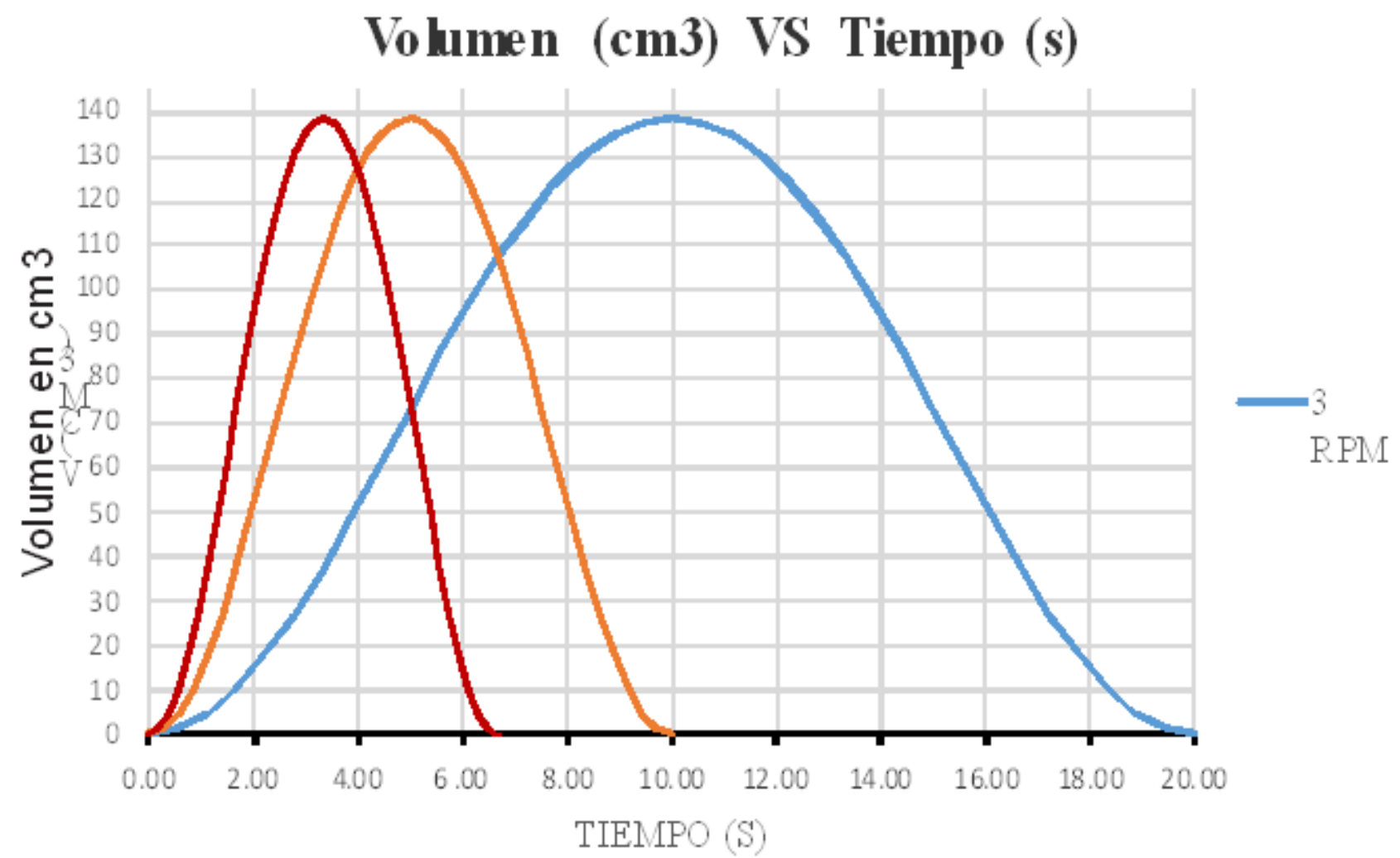

Figura 14. Variaciones en el volumen de agua dentro del cilindro de bombeo según el instante de análisis dentro del ciclo de succión y descarga de la bomba

El programa realizado para obtener los presentes resultados (aplicación vectorial en Geogebra) cuenta con un deslizador el cual controla los ángulos de rotación de la manivela, expresada por la linea verde en las Fig 8-12, es decir el ángulo “ $\alpha$ ”. Fonseca, G. 2015, realiza esta simulación de una bomba reciprocante (distinta a la bomba del presente trabajo) pero lo hace con varios deslizadores. Esto seria una programación mas compleja pero de mayor precisión. Nosotros controlamos velocidades y la asumimos como constantes mientras que Fonseca puede controlar el análisis hasta las variantes de las velocidades de rotación.

Se observa en las gráficas de la programación en vectores que los intervalos de las posiciones angulares son cada $60^{\circ}$ con lo que se aprecian 5 gráficas, siendo los círculos de la izquierda las gráficas de posición de la manivela y las gráficas de la derecha correspondientes a sus soluciones vectoriales, estas gráficas están hechas solo para una velocidad angular de 3 RPM, esto se debe por la limitación de espacio en el paper, si se representaran todos los resultados finales de esta programación se tendría 36 gráficas para 3 RPM, 36 gráficas para 6 RPM y 36 gráficas adicionales para 9 RPM, las cuales son imposible de abarcar en su totalidad

En el análisis vectorial (Geogebra) solo se muestran las magnitudes de los vectores y el sentido de los vectores están dados por la gráfica en el triángulo de velocidades (al costado de los valores) es por eso que no se encuentran signos en estos valores, pero en las Tablas de resultados si se observa valores con signo positivo y negativo, esto es porque en las Tablas se asigna un signo ( $\left.+\mathrm{o}_{-}^{-}\right)$a las direcciones vectoriales en la carrera del pistón, esto es que los signos positivos ocurre cuando el pistón esta en carrera ascendente dentro del cilindro, lo que realizaría la succión del agua, y cuando baja el pistón, la velocidad tendría signo negativo con lo que se estaría realizando la descarga de agua.

\section{Conclusiones}

Se demuestra que aunque las velocidades de rotación en la manivela sean constantes, estas se transforman en velocidades variables lineales en el pistón, con lo que no se puede hablar de un solo caudal en todo el proceso de bombeo de una bomba eólica reciprocante, si no que es un conjunto de caudales correspondiente a cada punto de análisis dentro del ciclo de succión y descarga.

Los promedios de caudales ofrecidos por la bomba reciprocante se pueden obtener de dos maneras; primero, realizando la función de promedio estadístico con los datos obtenidos por el análisis sistemático, este promedio nos da $10.449 \mathrm{~mm}^{3} / \mathrm{seg}$ para condiciones de movimiento de la manivela de 3 RPM, pero si hacemos una prueba de llenado de agua en 20 segundos y y el volumen del cilindro lo dividimos entre este tiempo, obtendremos $13.873 \mathrm{~mm}^{3} / \mathrm{seg}$ con lo cual se tendrá que aceptar el de los datos estadísticos, por ser mas exactos, y conservadores. Lo mismo se obtiene para los datos correspondientes al movimiento rotatorio de la manivela de 6 y 9 RPM 
Se concluye que, en la gráficas de Caudal vs Angulo de posición de la manivela, las curvas aparecen en forma proporcional, es decir que mientras mas RPM se tenga como movimiento de la manivela mayores caudales se obtiene en la producción del transporte de agua, lo cual difiere de las graficas de caudal vs tiempo, en el cual se tiene que a mayores RPM se tenga, menor es el tiempo que se necesita para la producción de un mismo caudal.

En las gráficas de volumen de agua versus tiempo se observa que no es una curva con pendiente constante, lo que concluye que las variaciones de agua dentro del cilindro de succión y descarga son variables lo cual respalda la idea de caudales variables por cada punto de tiempo y Ángulo de rotación de la manivela.

\section{Agradecimientos}

El autor agradece a la Universidad Nacional Agraria La Molina por proporcionar la Bomba eólica diseñado en sus instalaciones, la cual se digitalizo en 3D y se obtuvo todos los datos geométricos y de diseño necesarios para el análisis. Así mismo agradece a la Pontificia Universidad Católica del Perú, por su colaboración, brindándome sus laboratorios CAD-CAM de la facultad de Ingeniería Mecánica, para la digitalización de la bomba en 3D así como la ejecución de las pruebas en simulación de movimiento del mecanismo a escala real y en tiempo real. Alos docentes de la escuela de post grado de la facultad de Ingeniería Mecánica de la PUCP, por sus sabias enseñanzas que incidieron en mi formación profesional de la maestría

\section{6. literatura citada}

Batista C. 2014. Método sistemático. Disponible en: https://prezi.com/hymvvvoqmwh_/metodosistematico/.

Beer, F. ; Johnston, E. 2010. Mecánica vectorial para ingenieros. Dinámica. Editorial Mac Graw Hill, México

Erdman, A.; Sandor, G. 1996. Diseño de mecanismos Editorial Prentice Hall. México.

Fonseca, G., Hurtado, M. y Gutierrez, R. 2015 Bomba reciprocante. En Memorias del Encuentro de Clubes de Geogebra del Estado de Zulia, (pp 99-105). Venezuela.

Hibbeler, R. 2010. Mecánica vectorial para ingenieros II. Editorial Prentice Hall, México.

Meriam, J. 1995. Mecánica para ingenieros. Editorial Reverté S.A. Barcelona, España.

Mabie, H.; Ocvirk, F. 1978. Mecanismos y dinámica de maquinaria. Editorial Limusa. México.

Mejia, K. 1986. Diseño de un oleoducto secundario desde un campo petrolero hasta la estación de recolección y bombeo. Tesis. Recuperado de: http//ap.www.dspace. espol.edu.ae. 